\title{
Optimal Resource Control of Multi-Processor Multi- Radio Nodes using semi-Markov Decision Processes
}

\author{
Muhammad Tahir* and Ronan Farrell ${ }^{\dagger}$ \\ * Department of Electrical Engineering \\ University of Engineering and Technology Lahore, Pakistan \\ $\dagger$ Institute of Microelectronics and Wireless Systems \\ National University of Ireland Maynooth, Ireland \\ mtahir@uet.edu.pk, rfarrell@eeng.nuim.ie
}

\begin{abstract}
An optimal resource control mechanism for a multiprocessor, multi-radio node architecture is proposed. To achieve our objective, a constrained semi-Markov decision problem is formulated, which not only provides optimal resource control but also meets quality of service demands imposed by application. For each sensed event, the desired energy efficiency and performance tradeoff is achieved by the optimal stochastic policy, which selects an appropriate set of processors and radios. The proposed solution is assessed using the reported energy consumption measurements for the existing platforms. Performance evaluation results point towards the importance of proper pairing of processor and radio in achieving the energy efficiency and performance tradeoff. The proposed solution can also be employed for hand held devices equipped with multiple processors and radios.
\end{abstract}

\section{INTRODUCTION}

The emergence of a variety of multi-modal sensor platforms that span the spectra of cost, resolution, form-factor as well as functionality has opened avenues to meet the challenges of demanding applications. For instance, visual sensors range from expensive pan-tilt-zoom high-resolution digital cameras to inexpensive webcams or even cheaper cell-phone cameras. A node integrating these sensors with the an appropriate set of processors and communication interfaces can meet the demands of applications, which perform a dynamic set of operations ranging from simple motion sensing to complex video/image and/or audio processing tasks as well as their timely reporting. A closer look at the power consumption of different building blocks of these node architectures and an appropriate utilization of available resources can improve the energy efficiency while meeting performance requirements.

Microprocessors such as TIs MSP430 and Atmels AVR128 have low power consumptions of $3 \mathrm{~mW}$ and $33 \mathrm{~mW}$, respectively, but have a high energy consumption rate of $0.934 \mathrm{~mJ} / \mathrm{bit}$ [1]. In contrast, processors with more computational power such as Intel PXA255 used in SensEye and LEAP sensor nodes [2], [1], have a lower energy consumption rate of $0.046 \mathrm{~mJ} / \mathrm{bit}$. The communication interfaces also show a similar trend with the TI's CC2420 low data rate IEEE 802.15.4 compliant transceiver requires $979 n \mathrm{n} /$ bit while a higher-end IEEE 802.11 RF interface consumes only 112nJ/bit [1]. The high-end computation and communication devices, however, exhibit larger startup costs compared to low-end counterparts. Exploiting these characteristics in node architectures have resulted in considerable performance improvements [3], [4].

Energy efficiency improvement for complex architectures exploiting the hardware design are discussed in [1], [5]. A multi-processor node architecture is proposed in [1], which integrates fine-grained energy dissipation monitoring and sophisticated power control scheduling for different node subsystems. An experimental study of multi-radio hardware platform, using two of the most widely employed radio interfaces based on IEEE 802.15.4 and IEEE 802.11 is detailed in [5], showing that a proper processor-radio pairing can be crucial to exploit the energy efficiency of higher bandwidth radios. Sensor node platforms with multi-processor, multi-radio combinations are also developed [1], [3] and [4]. The authors in [4] show the significance of large dynamic range of power and performance for node lifetime. Different hardware modules with varying power and performance characteristics are configured as a microsensor system for various applications. A reconfigurable sensor node architecture with dual CPU and radios is developed in [1]. However, the hardware configuration is limited to the static interconnect. In [3], a sensor node platform with multiple CPUs and radios with a flexible interconnect is proposed. These solutions are limited to specific platforms and do not provide an analytical tool, which can be used to compare the energy efficiency across the node platforms.

Node lifetime analysis is performed in [6], where the authors have proposed an analytical lifetime performance model for trigger-driven and schedule driven node operation. However, the platform architecture in [6], is limited to a node architecture with single CPU-radio pair. The work in [6] is extended to a node architecture equipped with dual CPUs and radios in [7]. The extended analytical lifetime performance model in [7] can be used for a number of platforms with dual processors and radios. But using an objective to only minimize the energy consumption, without taking into account the benefit of using high-end processor/radio, limits the applicability of the proposed solution. In addition, the model is limited in its applicability, since it does not make any provisions for minimum quality of service (QoS) guarantees, often required by the applications. This may lead to the trivial solution of using only the low end processor/radio all the time. To get around these limitations, we have developed a performance model and an optimal resource control mechanism for a multi-modal 
sensor node architecture, equipped with multiple radios and processors. The model enables bandwidth QoS provisioning and is flexible to accommodate other application performance demands. Our work also differs from the dual processor/radio architecture of [7] by using a generalized objective function, which not only takes into account the power consumption cost of processing and communication but also renders the reward proportional to the bandwidth and processing power provided.

The contribution of this work is to provide an optimal resource control mechanism for a multi-processor, multi-radio architecture by solving a stochastic decision problem using semi-Markov decision model. The model provides an optimal policy to process and communicate sensed data. The proposed solution can be used for many hand-held devices with multiple radios/processors to achieve optimal performance.

\section{System Architecture AND Model}

We consider a multi-modal sensor node architecture with three vastly different processors and communication interfaces as shown in Fig. 1(a). The three processing units consists of an 8-bit minimal performance processor $\mathrm{P}_{0}$, a 16-bit moderate performance processor $\mathrm{P}_{1}$ and a 32-bit high performance processor $\mathrm{P}_{2}$. The processor $\mathrm{P}_{0}$ is interfaced to $\mathrm{P}_{1}$ and $\mathrm{P}_{2}$ directly using a standard serial bus. The data communication interface employs two short range radios, $R_{1}$ and $R_{2}$, based on IEEE 802.15.4 and IEEE 802.11b transceiver chips and a long range $3.5 \mathrm{G}$ wireless data module, $\mathrm{R}_{3}$, used for cellular applications. The processors $\mathrm{P}_{1}$ and $\mathrm{P}_{2}$ are interfaced to the radios through a reconfigurable interconnect. This architecture integrates a unique set of features in terms of processing power and data communication interface to meet the challenges of many demanding applications.

The node operates in trigger driven mode where each sensor event from the sensors (e.g. PIR, magnetic, acoustic etc.) connected to the processor $\mathrm{P}_{0}$ can wakeup either $\mathrm{P}_{1}$ or $\mathrm{P}_{2}$ for further data capturing and processing using their local sensors (e.g. cameras). The information after processing an event can either be communicated using one of the radios or it can be discarded as a false alarm. For each event, there is a sequence of operations starting at $\mathrm{P}_{0}$ followed by processing at either $\mathrm{P}_{1}$ or $\mathrm{P}_{2}$ and finally either communicating the processed data using one of the radios or discarding it as a false alarm. This sequence of operations and the associated choices made while performing those operations, can be represented formally using a state transition diagram. This is done by modeling the sequence of operations as a set $S=\left\{s_{i}, s_{p}, s_{c}, s_{t}\right\}$, where $s_{i}$ represent the idle state, $s_{p}$ is the processing state and $s_{c}$ represent the communication state and are shown in Fig. 1(b). The termination state, $s_{t}$, is effectively state $s_{i}$ and can be considered as an auxiliary state introduced to form one complete cycle of node operation. Fig. 1(b) also shows the transition probability and decision (action) pair for each state transition, which will be explained in Section III. In developing our model we have made the following assumptions:

- The accumulated event arrivals from all the sensors follow Poisson distribution with mean arrival rate $\lambda$.

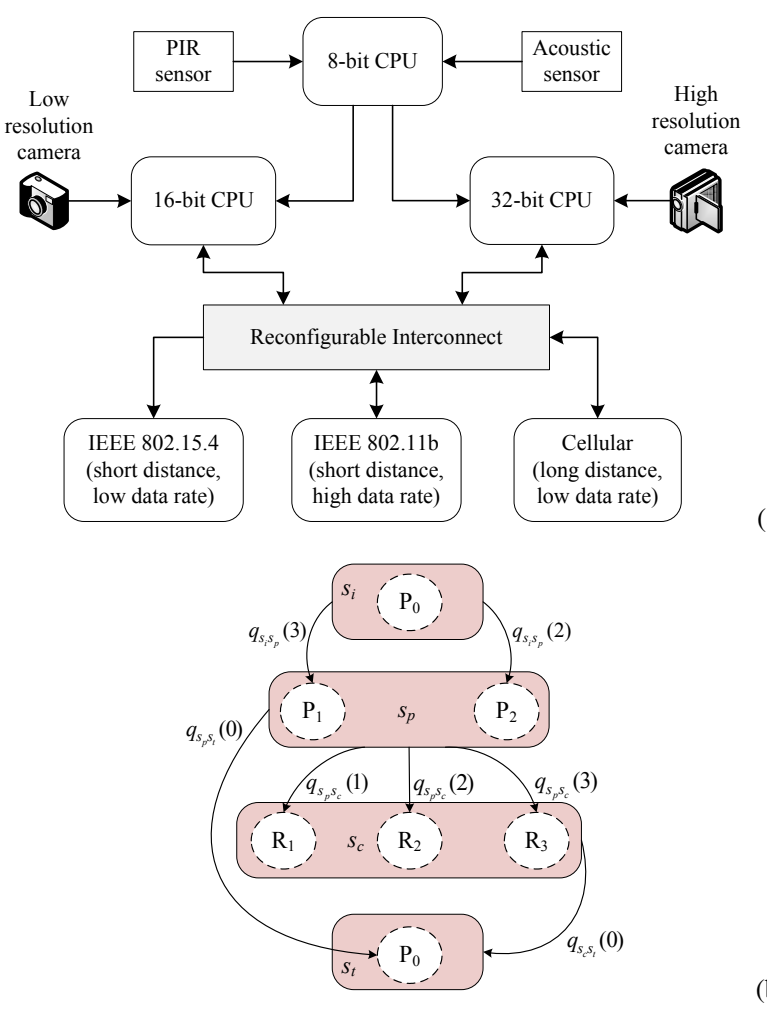

Fig. 1. State transition diagram for multi-tier node architecture. State transition probabilities along with the associated actions are also marked.

- The time duration a node spends in each processing and communication state is independent and identically distributed with arbitrary distribution.

- The mean holding time at processing and communication states is small compared to event inter-arrival times.

The first two assumptions mean that the state transitions can be modeled as a semi-Markov processes. The last assumption is needed for the validity of second assumption. The state transition diagram in Fig. 1(b) satisfies the Markov property, since the presence in state $s_{j} \in S$, only depends on the previous state and the action taken in that state and is independent of the prior state visits. On the other hand, since the time spent in each state is assumed to be random, the embedded chain associated with the state transition diagram of Fig. 1(b) is a semi-Markov chain. As a result the problem of optimal energy efficiency and performance tradeoff can be solved as a semiMarkov decision problem (SMDP).

\section{Energy EfFiciency and Performance Tradeoff FRAMEWORK}

In this section we first give details of building blocks, which are integrated to form the semi-Markov decision framework providing optimal tradeoff between energy efficiency and performance for multi-processor, multi-radio architecture.

\section{A. Action Space}

For each event arrival at $\mathrm{P}_{0}$ as shown in Fig. 1(a), the processor $\mathrm{P}_{0}$ is required to take an action to wake up either 
$\mathrm{P}_{1}$ or $\mathrm{P}_{2}$ for further processing and a possible communication of this event. Once sensor data is processed at either $\mathrm{P}_{1}$ or $\mathrm{P}_{2}$, a decision is made about its transmission and an action is taken by the corresponding processor. Let $A$ denote the set of all control actions and $A\left(s_{j}\right)$ is the set of control actions possible in state $s_{j}$ then control action $a_{k} \in A\left(s_{j}\right) \subseteq A$, at discrete time instant $k$, can have following values:

$$
a_{k}=\left\{\begin{array}{ll}
0 & \text { select } \mathrm{P}_{0} \\
1 & \text { select } \mathrm{P}_{1} \text { or } \mathrm{R}_{1} \\
2 & \text { select } \mathrm{P}_{2} \text { or } \mathrm{R}_{2} \\
3 & \text { select } \mathrm{R}_{3}
\end{array} .\right.
$$

To simplify the notation we will use $a$ for $a_{k}$ whenever appropriate. For $a=1(a=2)$ the choice between $\mathrm{P}_{1}$ and $\mathrm{R}_{1}\left(\mathrm{P}_{2}\right.$ and $\left.\mathrm{R}_{2}\right)$ depends on the current state. For instance, in state $s_{i}$, action $a=1$ will result in using processor $\mathrm{P}_{1}$, while in state $s_{p}$, action $a=1$ will result in using radio $\mathrm{R}_{1}$. It should be noted that all actions are not possible in each state. For instance, irrespective of current time instant, the state $s_{i}$ has only two possible actions i.e. $A\left(s_{i}\right)=\{1,2\}$, while for state $s_{p}$ we have $A\left(s_{p}\right)=\{0,1,2,3\}$.

\section{B. State Dynamics, Policy and Cost Function}

The state dynamics of the system can be characterized using the state transition probabilities, $q_{s_{j} s_{m}}(a)$, of the embedded chain and the expected sojourn time $\tau_{s_{j}}(a)$ for each state $s_{j}$ when action $a$ is chosen. Different state transition probabilities, also depicted for some states in Fig. 1(b), are given by:

$$
q_{s_{j} s_{m}}(a)=\left\{\begin{array}{cl}
1 & j=i, m=p, a \in\{1,2\} \\
p_{s_{j} s_{m}} & j=p, m=t, a=0 \\
p_{s_{j} s_{m}} & j=p, m=c, a \in\{1,2,3\} \\
1 & j=c, m=t, a=0 \\
1 & j=t, m=i, a=0 \\
0 & \text { otherwise }
\end{array},\right.
$$

where $p_{s_{j} s_{m}}$ is the probability of discarding an event due to false alarm. The expected sojourn time $\tau_{s_{j}}(a)$ when action $a$ is chosen in current state $s_{j}$ is given by

$$
\tau_{s_{j}}(a)=\sum_{s_{m}} p_{s_{j} s_{m}} \int_{0}^{\infty} t d F_{s_{j} s_{m}}(t \mid a)
$$

In (3), $d F_{s_{j} s_{m}}(t \mid a)$ is conditional cumulative distribution of transition time from $s_{j}$ to $s_{m}$ at time $t$ when action $a$ is chosen.

For each state $s_{j} \in S$ an action $a \in A\left(s_{j}\right)$ is chosen according to a policy $u_{s_{j}} \in \mathcal{U}$, where $\mathcal{U}$ is a set of admissible policies and is defined as

$$
\mathcal{U}=\left\{u: S \rightarrow A \mid u_{s_{j}} \in A\left(s_{j}\right), \forall s_{j} \in S\right\} .
$$

The semi-Markov decision framework selects a sequence of processing and communication states that results in an optimal tradeoff between energy consumption cost incurred and the resulting performance reward earned along the path from state $s_{i}$ to state $s_{t}$. Each state transition from $s_{j}$ to $s_{m}$ when action $a$ is taken, incurs an immediate fixed cost $C_{s_{j} s_{m}}(a)$ to account for startup and a cost rate of $c_{s_{j} s_{m}}(a)$ to accumulate the power consumption in state $s_{j}$ till the next action is taken. These cost components are used to obtain the mean expected cost for action $a$ in state $s_{j}$ as

$$
\begin{aligned}
\bar{C}_{s_{j}}(a)+\bar{c}_{s_{j}}(a) \tau_{s_{j}}(a)= & \sum_{s_{m}} p_{s_{j} s_{m}}\left(C_{s_{j} s_{m}}(a)\right. \\
& \left.+c_{s_{j} s_{m}}(a) \tau_{s_{j}}(a)\right) .
\end{aligned}
$$

To account for performance benefit, we use the reward rate, $\bar{r}_{s_{j}}(a)$, earned for each state action pair. For different processors the reward rate is the product of the processing power (in number of million-instructions executed per second i.e. (MIPS)) and the word size of the processor (e.g. 8-bit, 16-bit or 32-bit). For the communication interface the reward rate is product of the data transmission rate and the approximate maximum distance between the transmitter and receiver for a given packet loss rate. For the average cost-reward criterion selected as a measure of energy efficiency and performance tradeoff, the overall objective function, $J_{u}$ for any policy $u \in \mathcal{U}$ and an arbitrary initial state, is given by

$$
J_{u}=\lim _{K \rightarrow \infty} \frac{E_{u}\left\{\sum_{0 \leq k \leq K}\left(e_{k}-\beta \bar{r}_{s_{j}}\left(a_{k}\right)\right)\right\}}{E_{u}\left\{\sum_{0 \leq k \leq K} \tau_{s_{j}}\left(a_{k}\right)\right\}} .
$$

In (6), $e_{k}=\bar{C}_{s_{j}}\left(a_{k}\right)+\bar{c}_{s_{j}}\left(a_{k}\right) \tau_{s_{j}}\left(a_{k}\right), E_{u}$ is expectation under stationary policy $u$ and $\beta$ is user defined scaling parameter to achieve the tradeoff between relative average cost and corresponding reward earned. The aim is to find an optimal policy $u^{*} \in \mathcal{U}$ that minimizes $J_{u}$ for any arbitrary initial state.

\section{Problem Formulation}

The policy, which provides an optimal tradeoff between energy efficiency and performance of multi-processor multiradio architecture, is obtained by solving the following linear program formulation of semi-Markov decision problem:

$$
\begin{gathered}
\operatorname{minimize} \sum_{s_{j} \in S} \sum_{a \in A\left(s_{j}\right)}\left\{\bar{C}_{s_{j}}(a) \pi_{s_{j} a}+\bar{c}_{s_{j}}(a) \tau_{s_{j}}(a) \pi_{s_{j} a}\right. \\
\left.-\beta_{k} \bar{r}_{s_{j}}(a) \tau_{s_{j}}(a) \pi_{s_{i} a}\right\}
\end{gathered}
$$

subject to

$$
\begin{aligned}
& \sum_{a \in A\left(s_{m}\right)} \pi_{s_{m} a}-\sum_{s_{j} \in S} \sum_{a \in A\left(s_{j}\right)} q_{s_{j} s_{m}}(a) \pi_{s_{j} a}=0, s_{m} \in S, \\
& \sum_{s_{j} \in S} \sum_{a \in A\left(s_{j}\right)} \tau_{s_{j}}(a) \pi_{s_{j} a}=1,0 \leq \pi_{s_{j} a} .
\end{aligned}
$$

In (7), $\pi_{s_{j} a}$ are the decision variables. The term $\tau_{s_{j}}(a) \pi_{s_{j} a}$ is effectively the steady state probability of the system being in state $s_{j}$ and action $a$ is chosen. The first constraint in (7) represent the balance equations requiring that for any state $s_{m} \in S$, the long-run average number of transitions from state $s_{m}$ per time unit must be equal to the long-run average number of transitions into state $s_{m}$ per time unit. The second constraint guarantees that the sum of the steady state probabilities is one. Choosing linear programming formulation for solving the SMDP allows us to introduce minimum performance constraints, which is not possible when employing policy- or value-iteration methods to solve the SMDP. 


\section{Constraints}

Depending on the relative values of processing and communication costs as well as the corresponding rewards earned, the SMDP problem formulation in (7) can lead to a trivial solution of not making any data transmissions at all. To get around this problem we introduce the minimum data transmission constraint by requiring a certain percentage of events at $s_{i}$ to be communicated. Incorporating minimum data transmission constraint, the resource optimization problem in (7) becomes:

$$
\begin{array}{r}
\operatorname{minimize} \sum_{s_{j} \in S} \sum_{a \in A\left(s_{j}\right)}\left\{\bar{C}_{s_{j}}(a) \pi_{s_{j} a}+\bar{c}_{s_{j}}(a) \tau_{s_{j}}(a) \pi_{s_{j} a}\right. \\
\left.-\beta_{k} \bar{r}_{s_{j}}(a) \tau_{s_{j}}(a) \pi_{s_{i} a}\right\} \\
R_{\text {min }} \leq \sum_{\begin{array}{c}
\left.a \in A\left(s_{j}\right)\right|_{s_{j}=s_{c}} \\
\text { and constraints in }
\end{array}} \tau_{s_{j}}(a) \pi_{s_{j} a},
\end{array}
$$

In (8), $R_{\min }$ is minimum data transmission rate required. Due to the introduction of minimum data transmission rate constraint, optimal policy obtained can be a randomized policy. In that case optimal action $a^{*} \in A\left(s_{j}\right)$ is chosen probabilistically using optimal randomized stationary policy given as

$$
u_{s_{j}}^{*}(a)=\left\{\begin{array}{cl}
\frac{\pi_{s_{j} a}^{*}}{\sum_{a} \pi_{s_{j} a}^{*}} & a \in A\left(s_{j}\right), s_{j} \in S_{0}, \\
\text { arbitrary } & \text { otherwise }
\end{array}\right.
$$

where $S_{0}=\left\{s_{j} \mid \sum_{a} \pi_{s_{j} a}^{*}>0\right\}$ meaning that same action may not be taken every time a state is visited. For this case the unichain assumption is essential for guaranteeing the existence of an optimal stationary randomized policy [8].

\section{RESULTS}

To evaluate the performance of proposed resource control mechanism we first select an appropriate set of costs and rewards for the objective function. The costs incurred by CPUs and communication interfaces are measured in terms of their startup energy $\left(\bar{C}_{s_{j}}(a)\right)$ and running power $\left(\bar{c}_{s_{j}}(a)\right)$ consumptions [7]. The startup and running costs for selected processors and radio transceivers are provided in Table I. The reward $\left(\bar{r}_{s_{j}}(a)\right)$ for processing in Table I is obtained by multiplying processor speed in MIPS with normalized word size (NWS), where normalization is done by 32-bit word length and for communication interface it is obtained by multiplying data transmission rate in Mbps and coverage in meters. This communication reward definition provides the basis for fair comparison of high data rate short range and low data rate long range interfaces. Mean event arrival rate is $\lambda$ and follows a Poisson distribution. Expected sojourn times for each state action pair are obtained for a data payload of $10^{4}$ bytes and are given in Table II. Corresponding state transition probabilities are also provided in Table II. We require that at least $5 \%$ of the events are communicated and parameter $R_{\min }$ is chosen accordingly.

To evaluate the performance of optimal policy we study the utilization of each processor and radio. The utilization of processor $\mathrm{P}_{1}$ is obtained from $\tau_{s_{p}}(1) \pi_{s_{p} 1}$ and is shown
TABLE I

COSTS AND REWARDS FOR PROCESSING AND COMMUNICATION

\begin{tabular}{lccc}
\hline Processing & PIC10F200 & TI-MSP430 & SPM PXA255 \\
\hline Wakeup cost & 0 & $.018 \mathrm{~mJ}$ & $2.2 \mathrm{~mJ}$ \\
Running cost & $.85 \mathrm{~mW}$ & $7.6 \mathrm{~mW}$ & $825 \mathrm{~mW}$ \\
Reward (MIPS-NWS) & .25 & 8 & 200 \\
\hline Communication & CC2420 & SMC2532 & UC864-E \\
\hline Wakeup cost & $0.035 \mathrm{~mJ}$ & $5 \mathrm{~mJ}$ & $12 \mathrm{~mJ}$ \\
Running cost & $57.4 \mathrm{~mW}$ & $920 \mathrm{~mW}$ & $2584 \mathrm{~mW}$ \\
Reward (Mbps-m) & 12.5 & 330 & 1344 \\
\hline
\end{tabular}

TABLE II

MEAN SOJOURN TIMES AND STATE TRANSITION PROBABILITIES

\begin{tabular}{lclc}
\hline Symbol & Value & Symbol & Value \\
\hline$\tau_{s_{p}}(1)$ & $200 \mathrm{~ms}$ & $\tau_{s_{c}}(1)$ & $660 \mathrm{~ms}$ \\
$\tau_{s_{p}}(2)$ & $5 \mathrm{~ms}$ & $\tau_{s_{c}}(2)$ & $16.6 \mathrm{~ms}$ \\
$\tau_{s_{i}}(0)$ & $1 / \lambda$ & $\tau_{s_{c}}(3)$ & $330 \mathrm{~ms}$ \\
$p_{s_{p} s_{c}}$ & $0.1 \sim 0.9$ & $p_{s_{p} s_{t}}$ & $1-p_{s_{p} s_{c}}$ \\
\hline
\end{tabular}

in Fig. 2(b) as a function of weighting coefficient $\beta$ for different values of event arrival rate, $\lambda$. The utilization of processor $\mathrm{P}_{1}$ is proportional to event arrival rate $\lambda$ to meet the communication requirement imposed by $R_{m i n}$. Comparing processor utilization in Fig. 2, we observe a load sharing between $\mathrm{P}_{0}$ and $\mathrm{P}_{1}$ or $\mathrm{P}_{2}$ in contrast to load switching between $\mathrm{P}_{1}$ and $\mathrm{P}_{2}$. The difference in reward rates for $\mathrm{P}_{1}$ and $\mathrm{P}_{2}$ (see Table I) is large enough to result in policy change, which leads to load switching between $\mathrm{P}_{1}$ and $\mathrm{P}_{2}$ due to the superior cost performance tradeoff for $\mathrm{P}_{2}$.

Percentage utilization of radios $R_{2}$ and $R_{3}$ is shown in Fig. 3. Optimal policy for communication interface does not utilize $\mathrm{R}_{1}$ at all, mainly due to its low data rate and smaller coverage resulting in minimal reward. A small percentage utilization of $\mathrm{R}_{2}$ is mainly due to its high data transmission rate. A change in policy to use $R_{3}$ instead of $R_{2}$ for $\beta>2$ is due to better reward rate of $R_{3}$ compared to that of $R_{2}$. A policy change for both processor and radio at approximately same value of $\beta$ provides an insight that a proper processorradio paring is important, also pointed out in [5].

By setting parameters $\beta$ and $R_{\text {min }}$ equal to zero we emulate the solution proposed in [7] and compare its policy output with that of this paper. The results in Table III show that the policy output for [7] leads no data transmission at all. It also uses the same processor pair all the time. On the other hand the proposed solution leads to a policy utilizing different processors and communication interfaces, as observed from Table III, to achieve performance and energy efficiency tradeoff. The proposed optimal policy solution also meets the minimum data transmission rate requirement, showing its effectiveness in meeting the application performance demands compared to the solution in [7] which does not do any data transmission.

\section{CONCLUSiOns}

We propose an optimal resource control mechanism for a multi-processor, multi-radio node architecture, while meeting the QoS demands of the application. This is achieved by formulating a semi-Markov decision problem for the complex node architecture. The solution to decision problem leads 


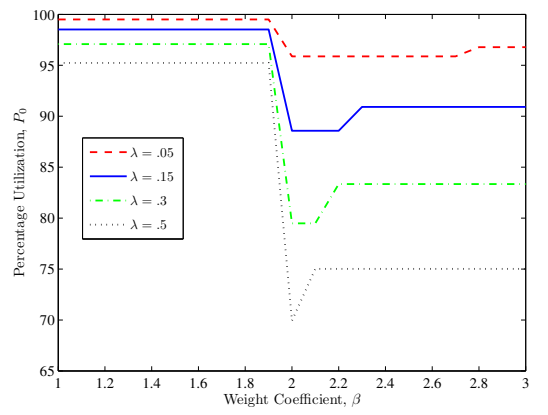

(a)

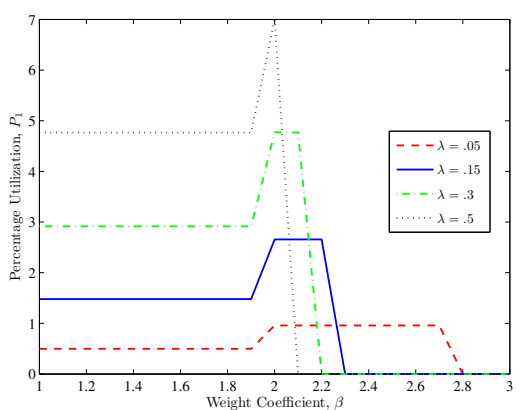

(b)

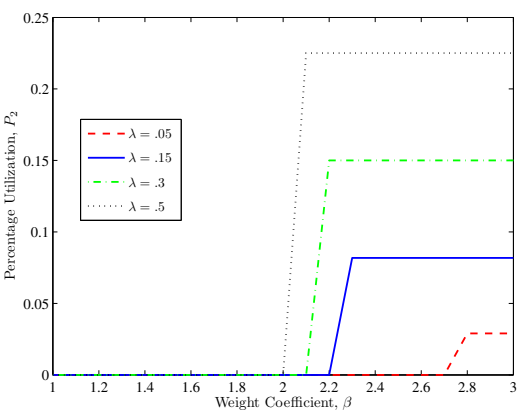

(c)

Fig. 2. Percentage utilization of (a) processor $P_{0}$, (b) processor $P_{1}$ and (c) processor $P_{2}$ as a function of weighting parameter $\beta$ for different values of $\lambda$.

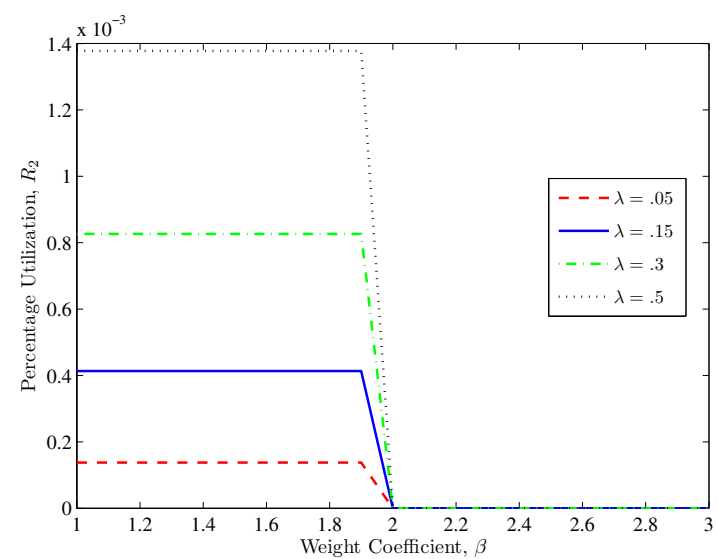

(a)

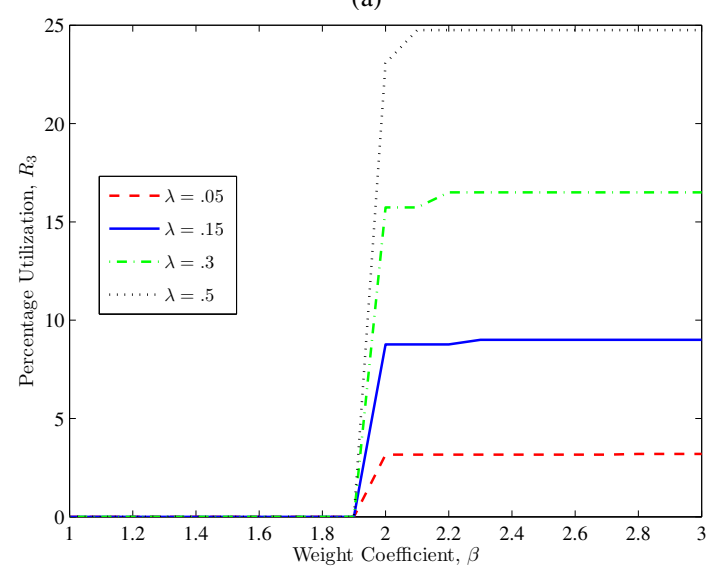

(b)

Fig. 3. Percentage utilization of (a) radio $\mathrm{R}_{2}$ and (b) radio $\mathrm{R}_{3}$ as a function of weighting parameter $\beta$ for different values of $\lambda$.

to an optimal energy efficiency and performance tradeoff and provides a stochastic policy to select the appropriate set of processors and communication interfaces to be used for each sensed event. Performance evaluation results reveal the importance of proper pairing of processor and radio.

The proposed solution is directly employable for different hand held devices equipped with multiple processors and radios. In addition, the proposed optimal resource control mechanism can be used for single processor multi-radio cell-
TABLE III

POLICY COMPARISON

\begin{tabular}{lcccccc}
\hline Policy $\backslash$ Utilization (\%) & $\mathrm{P}_{0}$ & $\mathrm{P}_{1}$ & $\mathrm{P}_{2}$ & $\mathrm{R}_{1}$ & $\mathrm{R}_{2}$ & $\mathrm{R}_{3}$ \\
\hline$[7], \lambda=.1, \beta=0$ & 99 & 1 & 0 & 0 & 0 & 0 \\
{$[7], \lambda=.5, \beta=0$} & 95 & 5 & 0 & 0 & 0 & 0 \\
{$[7], \lambda=1, \beta=0$} & 91 & 9 & 0 & 0 & 0 & 0 \\
Ours, $\lambda=.5, \beta=0$ & 95.23 & 4.76 & 0 & 0 & .0014 & 0 \\
Ours, $\lambda=.5, \beta=2$ & 70 & 7 & 0 & 0 & 0 & 23 \\
Ours, $\lambda=.5, \beta=3$ & 75 & 0 & .225 & 0 & 0 & 24.76 \\
\hline
\end{tabular}

phone architectures by decomposing the processing task into multiple states and assigning corresponding costs and rewards to each processing state.

\section{ACKNOWLEDGMENTS}

Research presented in this paper was funded by a Strategic Research Cluster grant (07/SRC/I1168) by Science Foundation Ireland under the National Development Plan. The authors gratefully acknowledge this support.

\section{REFERENCES}

[1] D. McIntire, K. Ho, B. Yip, A. Singh, W. Wu, and W. Kaiser, "The low power energy aware processing (leap) embedded networked sensor system," in International Symposium on Information Processing in Sensor Networks, IPSN, 2006, pp. 449-457.

[2] P. Kulkarni, D. Ganesan, P. Shenoy, and Q. Lu, "SensEye: a multitier camera sensor network," in Proceedings of the 13th annual ACM international conference on Multimedia, 2005, pp. 229-238.

[3] D. Lymberopoulos, N. Priyantha, and F. Zhao, "mPlatform: a reconfigurable architecture and efficient data sharing mechanism for modular sensor nodes," in International Symposium on Information Processing in Sensor Networks, IPSN, 2007, pp. 128-137.

[4] B. Schott, M. Bajura, J. Czarnaski, J. Flidr, T. Tho, and L. Wang, "A modular power-aware microsensor with $>1000 \mathrm{X}$ dynamic power range," in International Symposium on Information Processing in Sensor Networks, IPSN, 2005, pp. 469-474.

[5] D. Lymberopoulos, N. Priyantha, M. Goraczko, and F. Zhao, "Towards Energy Efficient Design of Multi-radio Platforms for Wireless Sensor Networks," in Information Processing in Sensor Networks, 2008. IPSN'08. International Conference on, 2008, pp. 257-268.

[6] D. Jung, T. Teixeira, A. Barton-Sweeney, and A. Savvides, "Model-based design exploration of wireless sensor node lifetimes," Lecture Notes in Computer Science, vol. 4373, pp. 277-292, 2007.

[7] D. Jung and A. Savvides, "An energy efficiency evaluation for sensor nodes with multiple processors, radios and sensors," in Proc. of IEEE Infocom, 2008, pp. 439-447.

[8] H. Tijms, A first course in stochastic models. John Wiley and Sons, 2003. 